\title{
MAPS PRESERVING PERIPHERAL SPECTRUM OF GENERALIZED JORDAN PRODUCTS OF OPERATORS
}

\author{
WEN ZHANG, JINCHUAN HOU, AND XIAOFEI QI
}

\begin{abstract}
Let $X_{1}$ and $X_{2}$ be complex Banach spaces with dimension at least three, $\mathcal{A}_{1}$ and $\mathcal{A}_{2}$ be standard operator algebras on $X_{1}$ and $X_{2}$, respectively. For $k \geq 2$, let $\left(i_{1}, \ldots, i_{m}\right)$ be a sequence with terms chosen from $\{1, \ldots, k\}$ and assume that at least one of the terms in $\left(i_{1}, \ldots, i_{m}\right)$ appears exactly once. Define the generalized Jordan product $T_{1} \circ T_{2} \circ \cdots \circ$ $T_{k}=T_{i_{1}} T_{i_{2}} \cdots T_{i_{m}}+T_{i_{m}} \cdots T_{i_{2}} T_{i_{1}}$ on elements in $\mathcal{A}_{i}$. This includes the usual Jordan product $A_{1} A_{2}+A_{2} A_{1}$, and the Jordan triple $A_{1} A_{2} A_{3}+A_{3} A_{2} A_{1}$. Let $\Phi: \mathcal{A} \rightarrow \mathcal{B}$ be a map with range containing all operators of rank at most three. It is shown that $\Phi$ satisfies that $\sigma_{\pi}\left(\Phi\left(A_{1}\right) \circ \cdots \circ \Phi\left(A_{k}\right)\right)=\sigma_{\pi}\left(A_{1} \circ \cdots \circ A_{k}\right)$ for all $A_{1}, \ldots, A_{k}$, where $\sigma_{\pi}(A)$ stands for the peripheral spectrum of $A$, if and only if $\Phi$ is a Jordan isomorphism multiplied by an $m$ th root of unity.
\end{abstract}

\section{INTRODUCTION}

There has been considerable interest in studying spectrum preserving maps on operator algebras in connection to the Kaplanskys problem on characterization of linear maps between Banach algebras preserving invertibility; see [1, 2, 8, 9, 20]. Early study focus on linear maps, additive maps, or multiplicative maps; see, e.g., [13]. Moreover, spectrum preserving maps on Banach algebras which are not assumed to be linear are studied by several authors (see [11, 12, 14, 16, 17, 18, 19]). In [14], Molnár characterized surjective maps $\Phi$ on bounded linear operators acting on a Hilbert space preserving the spectrum of the product of operators, i.e., $A B$ and $\Phi(A) \Phi(B)$ always have the same spectrum. Hou, Li and Wong [6, 7] studied respectively further the maps $\Phi$ between certain operator algebras preserving the spectrum of a generalized product $T_{1} * T_{2} * \cdots * T_{k}$ and a generalized Jordan product $T_{1} \circ T_{2} \circ \cdots \circ T_{k}$ of low rank operators.

Definition 1.1. Fix a positive integer $k$ and a finite sequence $\left(i_{1}, i_{2}, \ldots, i_{m}\right)$ such that $\left\{i_{1}, i_{2}, \ldots, i_{m}\right\}=\{1,2, \ldots, k\}$ and there is an $i_{p}$ not equal to $i_{q}$ for all other $q$. For operators

2010 Mathematical Subject Classification. 47B49, 47A12, 47L10.

Key words and phrases. Peripheral spectrum, generalized Jordan products, Banach spaces, standard operator algebras, preservers.

This work is partially supported by National Natural Science Foundation of China (No.11171249, 11101250, 11271217). 
$T_{1}, \ldots, T_{k}$, the operators

$$
T_{1} * T_{2} * \cdots * T_{k}=T_{i_{1}} T_{i_{2}} \cdots T_{i_{m}}
$$

and

$$
T_{1} \circ T_{2} \circ \cdots \circ T_{k}=T_{i_{1}} T_{i_{2}} \cdots T_{i_{m}}+T_{i_{m}} \cdots T_{i_{2}} T_{i_{1}}
$$

are respectively called generalized product and generalized Jordan product of $T_{1}, \ldots, T_{k}$.

Evidently, the generalized product $T_{1} * \cdots * T_{k}$ (the generalized Jordan product $T_{1} \circ \cdots \circ T_{k}$ ) covers the usual product $T_{1} T_{2}$ and the Jordan semi-triple product $T_{1} T_{2} T_{1}$ (the Jordan product $T_{1} T_{2}+T_{2} T_{1}$ and the Jordan triple product $T_{1} T_{2} T_{3}+T_{3} T_{2} T_{1}$ ), etc.. In [6] (resp. [7]) it was shown that, if $\Phi$ is a map between standard operator algebras with the range containing all operators of rank at most three, then, for all operators $T_{1}, T_{2}, \cdots, T_{k}$ of low rank, the spectra of $T_{1} * T_{2} * \cdots * T_{k}$ (resp., of $T_{1} \circ T_{2} \circ \cdots \circ T_{k}$ ) and of $\Phi\left(T_{1}\right) * \Phi\left(T_{2}\right) * \cdots * \Phi\left(T_{k}\right)$ (resp., of $\left.\Phi\left(T_{1}\right) \circ \Phi\left(T_{2}\right) \circ \cdots \circ \Phi\left(T_{k}\right)\right)$ are equal if and only $\Phi$ is a Jordan isomorphism multiplied by a $m$ th root of the unit.

Let $\mathcal{B}(X)$ be the Banach algebra of all bounded linear operators on a complex Banach space $X$. Denote by $\sigma(T)$ and $r(T)$ the spectrum and the spectral radius of $T \in \mathcal{B}(X)$, respectively. The peripheral spectrum of $T$ is defined by

$$
\sigma_{\pi}(T)=\{z \in \sigma(T):|z|=r(T)\}
$$

Since $\sigma(T)$ is compact, $\sigma_{\pi}(T)$ is a well-defined non-empty set and is an important spectral function. In [23], Tonev and Luttman studied maps preserving peripheral spectrum of the usual operator products on standard operator algebras. They studied also the corresponding problems in uniform algebras (see [11, 12]). Later Takeshi and Dai [22] generalized the result in [12] and characterized surjective maps $\phi$ and $\psi$ satisfying $\sigma_{\pi}(\phi(T) \psi(S))=\sigma_{\pi}(T S)$ on standard operator algebras. In [24] the maps preserving peripheral spectrum of Jordan semi-triple products of operators is characterized, and then, the maps preserving peripheral spectrum of generalized products of operators is also characterized in [25]. The maps preserving peripheral spectrum of Jordan products $A B+B A$ of operators on standard operator algebras are characterized by Cui and $\mathrm{Li}$ in 4 .

In this paper, we continue the study and characterize the maps preserving the peripheral spectrum of generalized Jordan products of operators between the standard operator algebras on complex Banach spaces. As expected, such maps are Jordan isomorphisms multiplied by a suitable root of the unit 1. However, the situation of generalized Jordan product is much more complicated than the Jordan product case. We can not use the similar technique for the 
case of Jordan product $A B+B A$ discussed in [4] to solve our problem. One of the reasons is that the root of $\Phi(I)$ may not be obtained.

In the following, let $X_{i}$ be a complex Banach space with dimension at least three and $\mathcal{A}_{i}$ the standard operator algebra on $X_{i}$, i.e., $\mathcal{A}_{i}$ contains all continuous finite rank operators on $X_{i}$, $i=1,2$. Note that a Jordan isomorphism $\Phi: \mathcal{A}_{1} \rightarrow \mathcal{A}_{2}$ is either an inner automorphism or anti-automorphism. In this case, it is obvious that $\sigma_{\pi}\left(\Phi\left(A_{1}\right) \circ \cdots \circ \Phi\left(A_{k}\right)\right)=\sigma_{\pi}\left(A_{1} \circ \cdots \circ A_{k}\right)$ holds for all $A_{1}, \ldots, A_{k}$. The main result of this paper is to show that the converse is also true.

Theorem 1.2. Let $\mathcal{A}_{i}$ be a standard operator algebra on a complex Banach space $X_{i}$ with $\operatorname{dim} X_{i} \geq 3, i=1,2$. Consider the product $T_{1} \circ \cdots \circ T_{k}$ defined in Definition 1.1. Let $\Phi: \mathcal{A}_{1} \rightarrow \mathcal{A}_{2}$ be a map with the range containing all operators in $\mathcal{A}_{2}$ of rank at most three. Then $\Phi$ satisfies

$$
\sigma_{\pi}\left(\Phi\left(A_{1}\right) \circ \cdots \circ \Phi\left(A_{k}\right)\right)=\sigma_{\pi}\left(A_{1} \circ \cdots \circ A_{k}\right)
$$

for any $A_{1}, \ldots, A_{k} \in \mathcal{A}_{1}$ if and only if one of the following conditions holds.

(1) There exist a scalar $\lambda \in \mathbb{C}$ with $\lambda^{m}=1$ and an invertible operator $T \in \mathcal{B}\left(X_{1}, X_{2}\right)$ such that $\Phi(A)=\lambda T A T^{-1}$ for all $A \in \mathcal{A}_{1}$.

(2) The spaces $X_{1}$ and $X_{2}$ are reflexive, and there exist a scalar $\lambda \in \mathbb{C}$ with $\lambda^{m}=1$ and an invertible operator $T \in \mathcal{B}\left(X_{1}^{*}, X_{2}\right)$ such that $\Phi(A)=\lambda T A^{*} T^{-1}$ for all $A \in \mathcal{A}_{1}$.

The "if" part is clear. So we need only to show the "only if" part of Theorem 1.2. This will be done in Section 3 .

\section{Characterizations of RANK ONE Operators}

To prove Theorem 1.2, it is important to characterize rank one operators in terms of the peripheral spectrum and generalized Jordan products.

Lemma 2.1. Let $X$ be a complex Banach space with $\operatorname{dim} X \geq 3$. Assume that $A \in \mathcal{B}(X)$ is a nonzero operator, $r$ and $s$ are integers with $s>r>0$. Then the following conditions are equivalent.

(1) A has rank one.

(2) $\sigma_{\pi}\left(B^{r} A B^{s}+B^{s} A B^{r}\right)$ has at most two elements for any $B \in \mathcal{B}(X)$.

(3) There does not exist an operator $B \in \mathcal{B}(X)$ with rank at most three such that $B^{r} A B^{s}+$ $B^{s} A B^{r}$ has rank three and $\sigma_{\pi}\left(B^{r} A B^{s}+B^{s} A B^{r}\right)$ has at most two elements.

Proof. The approach is similar to [7] but more complicated. We give details for reader's convenience.

The implications $(1) \Rightarrow(2) \Rightarrow(3)$ are clear. 
To prove $(3) \Rightarrow(1)$, suppose (3) holds but (1) is not true, i.e., $A$ has rank at least two.

If $A$ has rank at least 3 , then there are $x_{1}, x_{2}, x_{3} \in X$ such that $\left\{A x_{1}, A x_{2}, A x_{3}\right\}$ is linearly independent. Consider the operator matrix of $A$ on $\operatorname{span}\left\{x_{1}, x_{2}, x_{3}, A x_{1}, A x_{2}, A x_{3}\right\}$ and its complement:

$$
\left(\begin{array}{ll}
A_{11} & A_{12} \\
A_{21} & A_{22}
\end{array}\right) .
$$

Then $A_{11} \in M_{n}$ with $3 \leq n \leq 6$. By [6], there is a nonsingular $U$ on $\operatorname{span}\left\{x_{1}, x_{2}, x_{3}, A x_{1}\right.$, $\left.A x_{2}, A x_{3}\right\}$ such that $U^{-1} A_{11} U$ has an invertible 3-by-3 leading submatrix. We may further assume that the 3-by-3 matrix is in triangular form with nonzero diagonal entries $a_{1}, a_{2}, a_{3}$. Now let $B \in \mathcal{A}$ has operator matrix

$$
\left(\begin{array}{cc}
B_{11} & 0 \\
0 & 0
\end{array}\right),
$$

where $U B_{11} U^{-1}=\operatorname{diag}\left(1, b_{2}, b_{3}\right) \oplus 0_{n-3}$ with $B_{11}$ using the same basis as that of $A_{11}$ and $b_{2}, b_{3}$ being chosen such that $a_{1}, a_{2} b_{2}^{r+s}, a_{3} b_{3}^{r+s}$ are three distinct nonzero numbers with $\left|a_{1}\right|=$ $\left|a_{2} b_{2}^{r+s}\right|=\left|a_{3} b_{3}^{r+s}\right|$. It follows that $B^{r} A B^{s}+B^{s} A B^{r}$ has rank 3 and $\sigma_{\pi}\left(B^{r} A B^{s}+B^{s} A B^{r}\right)$ has three different points.

Next, suppose $A$ has rank 2. Choosing a suitable space decomposition of $X$, we may assume that $A$ has operator matrix $A_{1} \oplus 0$, where $A_{1}$ has one of the following forms.

$$
\text { (i) }\left(\begin{array}{lll}
a & 0 & b \\
0 & 0 & 0 \\
0 & 0 & c
\end{array}\right) \text {, (ii) }\left(\begin{array}{ccc}
a & 0 & 0 \\
0 & 0 & 1 \\
0 & 0 & 0
\end{array}\right) \text {, (iii) }\left(\begin{array}{ccc}
0 & 1 & 0 \\
0 & 0 & 1 \\
0 & 0 & 0
\end{array}\right) \text {, (iv) }\left(\begin{array}{cc}
0_{2} & I_{2} \\
0_{2} & 0_{2}
\end{array}\right) \text {. }
$$

If (i) holds, set $\theta=\frac{\pi}{2(r+s)}$. Then $\cos (r+s) \theta=0, \sin (r+s) \theta=1$ and $\sin (r-s) \theta \neq 0$. Let $d$ be a number such that $|a \sin (r-s) \theta|=\left|2 c d^{r+s}\right|$ but $2 c d^{r+s} \neq \pm i a \sin (r-s) \theta$. Let $B \in \mathcal{A}$ be represented by the operator matrix

$$
\left(\begin{array}{ccc}
\cos \theta & -\sin \theta & 0 \\
\sin \theta & \cos \theta & 0 \\
0 & 0 & d
\end{array}\right) \oplus 0
$$

Then $B^{r} A B^{s}+B^{s} A B^{r}$ has operator matrix

$$
\left(\begin{array}{ccc}
a \cos (r-s) \theta & -a & * \\
a & -\cos (r-s) \theta & * \\
0 & 0 & 2 c d^{r+s}
\end{array}\right) \oplus 0
$$

which has rank 3 and $\sigma_{\pi}\left(B^{r} A B^{s}+B^{s} A B^{r}\right)=\left\{2 c d^{r+s}, i a \sin (r-s) \theta,-i a \sin (r-s) \theta\right\}$. 
Suppose (ii) holds, set $\theta=\frac{\pi}{r+s}$. Then $\cos (r+s) \theta=-1, \sin (r+s) \theta=0$ and $\sin (r-s) \theta \neq 0$. Let $d$ be a number such that $|\sin (r-s) \theta|=\left|2 a d^{r+s}\right|$ but $2 a d^{r+s} \neq \pm i \sin (r-s) \theta$. Constructing $B$ by the operator matrix

$$
\left(\begin{array}{ccc}
d & 0 & 0 \\
0 & \cos \theta & -\sin \theta \\
0 & \sin \theta & \cos \theta
\end{array}\right) \oplus 0
$$

$B^{r} A B^{s}+B^{s} A B^{r}$ has operator matrix

$$
\left(\begin{array}{ccc}
2 a d^{r+s} & 0 & 0 \\
0 & 0 & \cos (r-s) \theta-1 \\
0 & \cos (r-s) \theta+1 & 0
\end{array}\right) \oplus 0
$$

which has rank 3 and $\sigma_{\pi}\left(B^{r} A B^{s}+B^{s} A B^{r}\right)=\left\{2 a d^{r+s}, i \sin (r-s) \theta,-i \sin (r-s) \theta\right\}$.

Suppose (iii) holds. First, assume that $s=2 r$. Let $B$ be such that $B^{r}$ has operator matrix

$$
\left(\begin{array}{lll}
0 & 1 & 0 \\
0 & 0 & 1 \\
1 & 0 & 0
\end{array}\right) \oplus 0
$$

Then $B^{r} A B^{s}+B^{s} A B^{r}$ has operator matrix

$$
\left(\begin{array}{lll}
0 & 1 & 0 \\
0 & 0 & 1 \\
2 & 0 & 0
\end{array}\right) \oplus 0
$$

which has rank 3 and $\sigma_{\pi}\left(B^{r} A B^{s}+B^{s} A B^{r}\right)=\left\{2^{1 / 3}, 2^{1 / 3} e^{i 2 \pi / 3}, 2^{1 / 3} e^{i 4 \pi / 3}\right\}$.

Next, suppose $s / r \neq 2$. Then $s>2$ and $2 r / s$ is not an integer. Let $\theta_{1}=2 \pi / s, \theta_{2}=4 \pi / s$. Then $1, e^{i r \theta_{1}}, e^{i r \theta_{2}}$ are distinct because $e^{i 4 \pi r / s}=e^{i 2 \pi(2 r / s)} \neq 1$ and $e^{i r \theta_{1}}=e^{i r \theta_{2}} / e^{i r \theta_{1}}=$ $e^{i 2 \pi r / s} \neq 1$. Thus, there exists an invertible $S \in M_{3}$ such that

$$
\left(\begin{array}{ccc}
1 & 0 & 0 \\
0 & e^{i r \theta_{1}} & 0 \\
0 & 0 & e^{i r \theta_{2}}
\end{array}\right)=S^{-1}\left(\begin{array}{ccc}
1 & \alpha & 0 \\
0 & e^{i r \theta_{1}} & 0 \\
m & 1 & e^{i r \theta_{2}}
\end{array}\right) S .
$$

Let $B$ have operator matrix

$$
S\left(\begin{array}{ccc}
1 & 0 & 0 \\
0 & e^{i \theta_{1}} & 0 \\
0 & 0 & e^{i \theta_{2}}
\end{array}\right) S^{-1} \oplus 0
$$


The operator matrix $B^{s}=I_{3} \oplus 0$ and the operator matrix of $B^{r}$ has the form

$$
S\left(\begin{array}{ccc}
1 & 0 & 0 \\
0 & e^{i r \theta_{1}} & 0 \\
0 & 0 & e^{i r \theta_{2}}
\end{array}\right) S^{-1} \oplus 0=\left(\begin{array}{ccc}
1 & \alpha & 0 \\
0 & e^{i r \theta_{1}} & 0 \\
m & 1 & e^{i r \theta_{2}}
\end{array}\right) \oplus 0
$$

Then $B^{r} A B^{s}+B^{s} A B^{r}=A B^{r}+B^{r} A$ has operator matrix

$$
\left(\begin{array}{ccc}
0 & 1+e^{i r \theta_{1}} & \alpha \\
m & 1 & e^{i r \theta_{1}}+e^{i r \theta_{2}} \\
0 & m & 1
\end{array}\right) \oplus 0
$$

which has rank 3. It follows from $\cos \frac{2 r \pi}{s}+1 \neq 0$ that $2 e^{i r \theta_{1}}+e^{i r \theta_{2}}+1=2\left(\cos \frac{2 r \pi}{s}+\right.$ $\left.i \sin \frac{2 r \pi}{s}\right)+\left(\cos \frac{4 r \pi}{s}+i \sin \frac{4 r \pi}{s}\right)+1=2 \cos \frac{2 r \pi}{s}\left(\cos \frac{2 r \pi}{s}+1\right)+i 2 \sin \frac{2 r \pi}{s}\left(\cos \frac{2 r \pi}{s}+1\right) \neq 0$. So let $m=\frac{-3}{2 e^{i r \theta_{1}}+e^{i r \theta_{2}}+1}$ and $\alpha=\frac{\left(2 e^{i r \theta_{1}}+e^{i r \theta_{2}}+1\right)\left(5+13 e^{i r \theta_{1}}+8 e^{i r \theta_{2}}\right)}{9}$, then $\sigma_{\pi}\left(B^{r} A B^{s}+B^{s} A B^{r}\right)=$ $\sigma_{\pi}\left(A B^{r}+B^{r} A\right)=\{2 i,-2 i, 2\}$.

If (iv) holds, then $X$ has dimension at least 4. We may use a different decomposition of $X$ and assume that $A$ has operator matrix

$$
\left(\begin{array}{ll}
0 & 1 \\
0 & 0
\end{array}\right) \oplus\left(\begin{array}{cc}
1 & 1 \\
-1 & -1
\end{array}\right) \oplus 0 .
$$

Let $\theta=\frac{\pi}{r+s}$. Then $\cos (r+s) \theta=-1, \sin (r+s) \theta=0$ and $\sin (r-s) \theta \neq 0$. Let $d$ be such that $|\sin (r-s) \theta|=\left|d^{r+s}\right|$ but $d^{r+s} \neq \pm i \sin (r-s) \theta$. Let $B \in \mathcal{A}$ be represented by the operator matrix

$$
\left(\begin{array}{ccc}
\cos \theta & -\sin \theta & 0 \\
\sin \theta & \cos \theta & 0 \\
0 & 0 & d
\end{array}\right) \oplus 0
$$

Then $B^{r} A B^{s}+B^{s} A B^{r}$ has operator matrix

$$
\left(\begin{array}{ccc}
0 & \cos (r-s) \theta+\cos (r+s) \theta & 0 \\
\cos (r-s) \theta-\cos (r+s) \theta & 0 & 0 \\
0 & 0 & d^{r+s}
\end{array}\right) \oplus 0
$$

which has rank 3 and $\sigma_{\pi}\left(B^{r} A B^{s}+B^{s} A B^{r}\right)=\left\{d^{r+s}, i \sin (r-s) \theta,-i \sin (r-s) \theta\right\}$.

Lemma 2.2. Suppose $s$ is a positive integer. Let $X$ be a complex Banach space with $\operatorname{dim} X \geq 3$. Let $A \in \mathcal{B}(X)$ be such that $A^{2} \neq 0$. Then the following conditions are equivalent.

(1) A has rank one.

(2) $\sigma_{\pi}\left(A B^{s}+B^{s} A\right)$ has at most two elements for any $B$ in $\mathcal{B}(X)$.

(3) $\sigma_{\pi}\left(A B^{s}+B^{s} A\right)$ has at most two elements for any $B$ whenever $\operatorname{rank} B \leq 3$ and $\operatorname{rank}\left(A B^{s}+\right.$ $\left.B^{s} A\right) \leq 3$. 
Proof. The implications $(1) \Rightarrow(2) \Rightarrow(3)$ are clear.

Suppose (3) holds but (1) is not true, i.e., $A$ has rank at least two such that $A^{2} \neq 0$.

First suppose $A$ has rank 2. Since $A^{2} \neq 0$, choosing a suitable space decomposition of $X$, we may assume that $A$ has operator matrix $A_{1} \oplus 0$, where $A_{1}$ has one of the following forms.

$$
\text { (i) }\left(\begin{array}{lll}
a & 0 & b \\
0 & 0 & 0 \\
0 & 0 & c
\end{array}\right) \text {, (ii) }\left(\begin{array}{ccc}
a & 0 & 0 \\
0 & 0 & 1 \\
0 & 0 & 0
\end{array}\right) \text {, (iii) }\left(\begin{array}{lll}
0 & 1 & 0 \\
0 & 0 & 1 \\
0 & 0 & 0
\end{array}\right) \text {. }
$$

If (i) holds, set $\theta=\frac{\pi}{2 s}$. Then $\cos s \theta=0, \sin s \theta=1$. Let $d$ be such that $|a|=\left|2 c d^{s}\right|$ but $2 c d^{s} \neq \pm i a$. Let $B \in \mathcal{A}$ be represented by the operator matrix

$$
\left(\begin{array}{ccc}
\cos \theta & -\sin \theta & 0 \\
\sin \theta & \cos \theta & 0 \\
0 & 0 & d
\end{array}\right) \oplus 0
$$

Then $A B^{s}+B^{s} A$ has operator matrix

$$
\left(\begin{array}{ccc}
0 & -a & * \\
a & 0 & * \\
0 & 0 & 2 c d^{s}
\end{array}\right) \oplus 0
$$

which implies that $\sigma_{\pi}\left(A B^{s}+B^{s} A\right)=\left\{2 c d^{s}, i a,-i a\right\}$.

Suppose (ii) holds. Since the matrix

$$
C=\left(\begin{array}{ccc}
1 / 2 & a & 0 \\
0 & 0 & 0 \\
-1 / 2 & 0 & -2
\end{array}\right)
$$

is similar to a matrix with distinct eigenvalues $0,1 / 2,-2$, there exists an operator $B$ of rank 2 such that the operator matrix of $B^{s}$ equals $C \oplus 0$. It follows that the operator matrix of $A B^{s}+B^{s} A$ is

$$
\left(\begin{array}{ccc}
a & a^{2} & a \\
-1 / 2 & 0 & -2 \\
-a / 2 & 0 & 0
\end{array}\right) \oplus 0
$$

and $\sigma_{\pi}\left(A B^{s}+B^{s} A\right)=\{a, i a,-i a\}$.

Suppose (iii) holds. Since the matrix

$$
C=\left(\begin{array}{lll}
0 & 1 & 0 \\
0 & 0 & 1 \\
1 & 0 & 0
\end{array}\right)
$$


has distinct eigenvalues $1, e^{i 2 \pi / 3}, e^{i 4 \pi / 3}$, there exists an operator $B$ of rank 3 such that the operator matrix of $B^{s}$ equals $C \oplus 0$. Then $A B^{s}+B^{s} A$ has operator matrix

$$
\left(\begin{array}{lll}
0 & 0 & 2 \\
1 & 0 & 0 \\
0 & 1 & 0
\end{array}\right) \oplus 0
$$

and $\sigma_{\pi}\left(A B^{s}+B^{s} A\right)=\left\{2^{1 / 3}, 2^{1 / 3} e^{i 2 \pi / 3}, 2^{1 / 3} e^{i 4 \pi / 3}\right\}$.

Now, suppose $A$ has rank at least 3 . Since $A^{2} \neq 0$, there is $x \in X$ such that $A^{2} x \neq 0$. We consider the following two cases.

Case 1. There is $x \in X$ such that $\left[x, A x, A^{2} x\right]$ has dimension 3 .

Decompose $X$ into $\left[x, A x, A^{2} x\right]$ and its complement. The operator matrix of $A$ has the form

$$
\left(\begin{array}{llll}
0 & 0 & c_{1} & * \\
1 & 0 & c_{2} & * \\
0 & 1 & c_{3} & * \\
0 & 0 & * & *
\end{array}\right)
$$

Subcase 1. $c_{1} \neq 0, c_{2}=0$.

Since the matrix

$$
C=\left(\begin{array}{lll}
1 & 0 & 0 \\
0 & 2 & 0 \\
0 & 0 & 0
\end{array}\right)
$$

has distinct eigenvalues $1,2,0$, there exists an operator $B$ of rank 2 such that the operator matrix of $B^{s}$ equals $C \oplus 0$. Then $A B^{s}+B^{s} A$ has operator matrix

$$
\left(\begin{array}{cccc}
0 & 0 & c_{1} & * \\
3 & 0 & 0 & * \\
0 & 2 & 0 & 0 \\
0 & 0 & 0 & 0
\end{array}\right) .
$$

Writing $6 c_{1}=r e^{i \theta}$, we have $\sigma_{\pi}\left(A B^{s}+B^{s} A\right)=\left\{r^{1 / 3} e^{i \theta / 3}, r^{1 / 3} e^{i(\theta+2 \pi) / 3}, r^{1 / 3} e^{i(\theta+4 \pi) / 3}\right\}$.

Subcase 2. $c_{1} \neq 0, c_{2} \neq 0, c_{3}=0$.

Let $\alpha \neq 0$ such that $\alpha^{2} c_{2}+1=0$, Since the matrix

$$
C=\left(\begin{array}{ccc}
\alpha & 1 & 0 \\
0 & -\alpha & -1 \\
0 & 0 & 0
\end{array}\right)
$$


has distinct eigenvalues $\alpha,-\alpha, 0$, there exists an operator $B$ of rank 2 such that the operator matrix of $B^{s}$ equals $C \oplus 0$. Then $A B^{s}+B^{s} A$ has operator matrix

$$
\left(\begin{array}{cccc}
1 & 0 & \alpha c_{1}+c_{2} & * \\
0 & 0 & -\alpha c_{2} & * \\
0 & -\alpha & -1 & 0 \\
0 & 0 & 0 & 0
\end{array}\right),
$$

and $\sigma_{\pi}\left(A B^{s}+B^{s} A\right)=\left\{1, e^{i 2 \pi / 3}, e^{i 4 \pi / 3}\right\}$.

Subcase 3. $c_{1} \neq 0, c_{2} \neq 0, c_{3} \neq 0$.

Let $\alpha \neq 0$ such that $3 \alpha c_{1}+4 c_{2}=0$. Since the matrix

$$
C=\left(\begin{array}{lll}
1 & 0 & 0 \\
\alpha & 2 & 0 \\
0 & 0 & 0
\end{array}\right)
$$

has distinct eigenvalues $1,2,0$, there exists an operator $B$ of rank 2 such that the operator matrix of $B^{s}$ equals $C \oplus 0$. Then $A B^{s}+B^{s} A$ has operator matrix

$$
\left(\begin{array}{cccc}
0 & 0 & c_{1} & * \\
3 & 0 & 2 c_{2}+\alpha c_{1} & * \\
\alpha & 2 & 0 & 0 \\
0 & 0 & 0 & 0
\end{array}\right) .
$$

With $6 c_{1}=r e^{i \theta}$, we see that $\sigma_{\pi}\left(A B^{s}+B^{s} A\right)=\left\{r^{1 / 3} e^{i \theta / 3}, r^{1 / 3} e^{i(\theta+2 \pi) / 3}, r^{1 / 3} e^{i(\theta+4 \pi) / 3}\right\}$.

Subcase 4. $c_{1}=0, c_{2} \neq 0, c_{3} \neq 0$.

Choose $\alpha \neq 0$ such that $\alpha^{2} c_{2}+\alpha c_{3}+1=0$. Since the matrix

$$
C=\left(\begin{array}{ccc}
\alpha & 1 & 0 \\
0 & -\alpha & -1 \\
0 & 0 & 0
\end{array}\right)
$$

has distinct eigenvalues $\alpha,-\alpha, 0$, there exists an operator $B$ of rank 2 such that the operator matrix of $B^{s}$ equals $C \oplus 0$. Then $A B^{s}+B^{s} A$ has operator matrix

$$
\left(\begin{array}{cccc}
1 & 0 & c_{2} & * \\
0 & 0 & -\alpha c_{2}-c_{3} & * \\
0 & -\alpha & -1 & 0 \\
0 & 0 & 0 & 0
\end{array}\right)
$$

and thus $\sigma_{\pi}\left(A B^{s}+B^{s} A\right)=\left\{1, e^{i 2 \pi / 3}, e^{i 4 \pi / 3}\right\}$.

Subcase 5. $c_{1}=0, c_{2}=0, c_{3} \neq 0$. 
Let $\alpha \neq 0$ such that $\alpha c_{3}+1=0$, Since the matrix

$$
C=\left(\begin{array}{ccc}
\alpha & 1 & 0 \\
0 & -\alpha & -1 \\
0 & 0 & 0
\end{array}\right)
$$

has distinct eigenvalues $\alpha,-\alpha, 0$, there exists an operator $B$ of rank 2 such that the operator matrix of $B^{s}$ equals $C \oplus 0$. Then $A B^{s}+B^{s} A$ has operator matrix

$$
\left(\begin{array}{cccc}
1 & 0 & 0 & * \\
0 & 0 & c_{3} & * \\
0 & -\alpha & -1 & 0 \\
0 & 0 & 0 & 0
\end{array}\right)
$$

and thus $\sigma_{\pi}\left(A B^{s}+B^{s} A\right)=\left\{1, e^{i 2 \pi / 3}, e^{i 4 \pi / 3}\right\}$.

Subcase 6. $c_{1}=0, c_{2} \neq 0, c_{3}=0$.

Pick $\alpha \neq 0$ such that $\alpha^{2} c_{2}+1=0$. Since the matrix

$$
C=\left(\begin{array}{ccc}
\alpha & 1 & 0 \\
0 & -\alpha & -1 \\
0 & 0 & 0
\end{array}\right)
$$

has distinct eigenvalues $\alpha,-\alpha, 0$, there exists an operator $B$ of rank 2 such that the operator matrix of $B^{s}$ equals $C \oplus 0$. Then $A B^{s}+B^{s} A$ has operator matrix

$$
\left(\begin{array}{cccc}
1 & 0 & c_{2} & * \\
0 & 0 & -\alpha c_{2} & * \\
0 & -\alpha & -1 & 0 \\
0 & 0 & 0 & 0
\end{array}\right)
$$

and $\sigma_{\pi}\left(A B^{s}+B^{s} A\right)=\left\{1, e^{i 2 \pi / 3}, e^{i 4 \pi / 3}\right\}$.

Subcase 7. $c_{1}=0, c_{2}=0, c_{3}=0$.

In this subcase we take

$$
C=\left(\begin{array}{ccc}
-1 & 1 & -1 \\
2 & 1 & -1 \\
0 & 0 & 0
\end{array}\right)
$$


which has distinct eigenvalues $\sqrt{3},-\sqrt{3}, 0$. Thus there exists an operator $B$ of rank 2 such that the operator matrix of $B^{s}$ equals $C \oplus 0$. Then $A B^{s}+B^{s} A$ has operator matrix

$$
\left(\begin{array}{cccc}
1 & -1 & 0 & * \\
0 & 0 & -1 & * \\
2 & 1 & -1 & 0 \\
0 & 0 & 0 & 0
\end{array}\right)
$$

with $\sigma_{\pi}\left(A B^{s}+B^{s} A\right)=\left\{3^{1 / 3}, 3^{1 / 3} e^{i 2 \pi / 3}, 3^{1 / 3} e^{i 4 \pi / 3}\right\}$.

Case 2. For every $x \in X,\left\{x, A x, A^{2} x\right\}$ is a linearly dependent set.

In this case $A$ is a locally algebraic operator, and hence a result due to Kaplansky (see, for example, [10]) tells us that $A$ is an algebraic operator of degree not greater than 2 . So there exist $\alpha, \beta, \gamma \in \mathbb{C}$ such that $\alpha A^{2}+\beta A+\gamma I=0$ with $(\alpha, \beta, \gamma) \neq(0,0,0)$.

If $\alpha=0$, then $\beta \neq 0$ and $\gamma \neq 0$, and therefore, $A$ is a scalar operator. Take $B=$ $\operatorname{diag}\left(1, \mathrm{e}^{\mathrm{i} 2 \pi / 3 \mathrm{~s}}, \mathrm{e}^{\mathrm{i} 4 \pi / 3 \mathrm{~s}}\right) \oplus 0$. Then $\sigma_{\pi}\left(A B^{s}+B^{s} A\right)$ contains three different points, a contradiction.

Now assume that $\alpha \neq 0$. Since $A^{2} \neq 0$, it follows that $(\beta, \gamma) \neq(0,0)$ and $\sigma(A)=\{a, b\}$, where $a, b \in \mathbb{C}$.

Subcase 1. $\sigma(A)=\{a, b\}$ with $a \neq 0$ and $b \neq 0$.

Then there exist linearly independent vectors $x_{1}, x_{2} \in X$ such that $A x_{1}=a x_{1}, A x_{2}=b x_{2}$. Since $A$ has rank at least 3 , there is $y \in X$ such that $y \notin\left[x_{1}, x_{2}\right]$ and $A y=a y$ or $A y=b y$. Then there is a decomposition of $X$ so that $A$ has operator matrix

$$
\left(\begin{array}{cc}
A_{0} & * \\
0 & *
\end{array}\right)
$$

where $A_{0}=\operatorname{diag}(\mathrm{a}, \mathrm{b}, \mathrm{a}) \operatorname{or} \operatorname{diag}(\mathrm{a}, \mathrm{b}, \mathrm{b})$. Then there is $B$ with operator matrix $B_{1} \oplus 0$, where $B_{1}=\operatorname{diag}\left(1, \mathrm{~b}_{1}, \mathrm{~b}_{2}\right)$, such that $A B^{s}+B^{s} A$ has operator matrix

$$
\left(\begin{array}{cc}
A_{0} B_{1}^{s}+B_{1}^{s} A_{0} & * \\
0 & 0
\end{array}\right)
$$

which has rank 3 and $\sigma_{\pi}\left(A B^{s}+B^{s} A\right)$ contains three different points.

Subcase 2. $\sigma(A)=\{a, 0\}$ with $a \neq 0$.

Then there exist linearly independent vectors $x_{1}, x_{2} \in X$ such that $A x_{1}=a x_{1}, A x_{2}=0$. Since $A$ has rank at least 3 , there is $y \in X$ such that $y \notin\left[x_{1}, x_{2}\right]$ and $A y=a y$. Then there is a decomposition of $X$ so that $A$ has operator matrix

$$
\left(\begin{array}{cc}
A_{0} & * \\
0 & *
\end{array}\right)
$$


where $A_{0}=\operatorname{diag}(\mathrm{a}, 0, \mathrm{a})$. In this case, we can use the argument in the proof when $A$ has rank 2 to choose $B$ with operator matrix $B_{1} \oplus 0$ so that $B_{1} \in M_{3}$ and $A B^{s}+B^{s} A$ has operator matrix

$$
\left(\begin{array}{cc}
A_{0} B_{1}^{s}+B_{1}^{s} A_{0} & * \\
0 & 0
\end{array}\right)
$$

which has rank 3 and $\sigma_{\pi}\left(A B^{s}+B^{s} A\right)$ contains three different points.

Corollary 2.3. Suppose $s$ is a positive integer. Let $X$ be a complex Banach space with $\operatorname{dim} X \geq 3$ and let $0 \neq A \in \mathcal{B}(X)$. Then the following conditions are equivalent.

(1) A has rank one, or $A$ has rank two with $A^{2}=0$.

(2) $\sigma_{\pi}\left(A B^{s}+B^{s} A\right)$ has at most two elements for any $B$ in $B(X)$.

(3) There exists no operator $B$ with rank at most 3 such that $B^{r} A B^{s}+B^{s} A B^{r}$ has rank at most 6 and $\sigma_{\pi}\left(B^{r} A B^{s}+B^{s} A B^{r}\right)$ has more than two elements.

Proof. (1) $\Rightarrow(2)$. If $A$ has rank one, then (2) clearly holds. If $A$ has rank two and $A^{2}=0$, then there is a decomposition of $X$ such that $A$ has operator matrix

$$
\left(\begin{array}{ccc}
0_{2} & I_{2} & 0 \\
0_{2} & 0_{2} & 0 \\
0 & 0 & 0
\end{array}\right)
$$

So, for any $B$ in $\mathcal{A}$ such that $B^{s}$ has operator matrix

$$
\left(\begin{array}{lll}
B_{11} & B_{12} & B_{13} \\
B_{21} & B_{22} & B_{23} \\
B_{31} & B_{32} & B_{33}
\end{array}\right)
$$

$A B^{s}+B^{s} A$ has operator matrix

$$
\left(\begin{array}{ccc}
B_{21} & B_{11}+B_{22} & B_{23} \\
0 & B_{21} & 0 \\
0 & B_{31} & 0
\end{array}\right)
$$

Then $\sigma_{\pi}\left(A B^{s}+B^{s} A\right)=\sigma_{\pi}\left(B_{21}\right)$ has at most two different elements as $B_{21} \in M_{2}$.

The implication $(2) \Rightarrow(3)$ is clear.

Finally, we verify the implication $(3) \Rightarrow(1)$. If (3) holds, by Lemma 2.2 , we see that $A$ is rank 1 whenever $A^{2} \neq 0$. If $A^{2}=0$, we claim that rankA $\leq 2$. If it is not true, then we can find linearly independent vectors $x_{1}, x_{2}, x_{3} \in X$ such that $\left\{A x_{1}, A x_{2}, A x_{3}\right\}$ is a linearly independent set. It follows from $A^{2}=0$ that $\left\{x_{1}, x_{2}, x_{3}, A x_{1}, A x_{2}, A x_{3}\right\}$ is a linearly independent set. Let $N$ be a closed subspace of $X$ such that $X=\operatorname{span}\left\{\mathrm{x}_{1}, \mathrm{x}_{2}, \mathrm{x}_{3}, \mathrm{Ax}_{1}, \mathrm{Ax}_{2}, \mathrm{Ax}_{3}\right\} \oplus \mathrm{N}$. 
Then $A$ has operator matrix

$$
\left(\begin{array}{ccc}
0_{3} & 0_{3} & * \\
I_{3} & 0_{3} & * \\
0 & 0 & *
\end{array}\right)
$$

Take

$$
B=\left(\begin{array}{cc}
D & D \\
0_{3} & 0_{3}
\end{array}\right) \oplus 0, \text { with } D=\operatorname{diag}\left(1, \mathrm{e}^{\mathrm{i} 2 \pi / 3 \mathrm{~s}}, \mathrm{e}^{\mathrm{i} 4 \pi / 3 \mathrm{~s}}\right)
$$

Then

$$
\sigma_{\pi}\left(A B^{s}+B^{s} A\right)=\left(\begin{array}{ccc}
C & 0_{3} & * \\
C & C & 0 \\
0 & 0 & 0
\end{array}\right) \text {, with } C=\operatorname{diag}\left(1, \mathrm{e}^{\mathrm{i} 2 \pi / 3}, \mathrm{e}^{\mathrm{i} 4 \pi / 3}\right),
$$

which has rank 6 and $\sigma_{\pi}\left(A B^{s}+B^{s} A\right)=\left\{1, e^{i 2 \pi / 3}, e^{i 4 \pi / 3}\right\}$.

The following lemma comes from [4].

Lemma 2.4. Let $x \in X$ and $f \in X^{*}$. Then, for every $B \in \mathcal{A}$,

$$
\sigma_{\pi}(B x \otimes f+x \otimes f B)= \begin{cases}\{f(B x)\} & \text { if } f(x)=0 \text { or } f\left(B^{2} x\right)=0, \\ \left\{ \pm \sqrt{f\left(B^{2} x\right) f(x)}\right\} & \text { if } f(x) \neq 0, f(B x)=0, f\left(B^{2} x\right) \neq 0 \\ \{\alpha\} & \text { if } f(x) \neq 0, f(B x) \neq 0, f\left(B^{2} x\right) \neq 0\end{cases}
$$

where the scalar

$$
|\alpha|=\max \left\{\left|f(B x)+\sqrt{f\left(B^{2} x\right) f(x)}\right|,\left|f(B x)-\sqrt{f\left(B^{2} x\right) f(x)}\right|\right\}
$$

\section{Proof of the MAIN RESUlt}

In this section we will complete the proof of Theorem 1.2.

It is clear that Theorem 1.2 follows from the special case below, by considering $A_{i_{p}}=A$ and all other $A_{i_{q}}=B$.

Theorem 3.1. Let $\mathcal{A}_{1}$ and $\mathcal{A}_{2}$ be standard operator algebras on complex Banach spaces $X_{1}$ and $X_{2}$, respectively. Assume that $\Phi: \mathcal{A}_{1} \rightarrow \mathcal{A}_{2}$ is a map, the range of which contains all operators of rank at most three. Suppose also that $\Phi$ satisfies

$$
\sigma_{\pi}\left(B^{r} A B^{s}+B^{s} A B^{r}\right)=\sigma_{\pi}\left(\Phi(B)^{r} \Phi(A) \Phi(B)^{s}+\Phi(B)^{s} \Phi(A) \Phi(B)^{r}\right)
$$

for all $A, B \in \mathcal{A}_{1}$. Then one of the two assertions in Theorem 1.2 holds with $m=r+s+1$.

Thus we focus our attention to prove Theorem 3.1. 
We note that the case when $s=r>0$ has been verified in [25]. So, unless specified otherwise, we will assume $s>r \geq 0$ in the rest of this section. In below, we first show that $\Phi$ in Theorem 3.1 is injective.

For a Banach space $X$, denote by $\mathcal{I}_{1}(X)$ the set of all rank one idempotent operators in $\mathcal{B}(X)$. In other words, $\mathcal{I}_{1}(X)$ consists of all bounded operators $x \otimes f$ with $x \in X, f \in X^{*}$ and $\langle x, f\rangle=f(x)=1$.

The following Lemma 3.2 was proved in [7].

Lemma 3.2. Let $A, A^{\prime} \in \mathcal{B}(X)$ for some Banach space $X$. Suppose

$$
\langle A x, f\rangle=0 \Leftrightarrow\left\langle A^{\prime} x, f\right\rangle=0, \quad \text { for all } x \otimes f \in \mathcal{I}_{1}(X) .
$$

Then $A^{\prime}=\lambda A$ for some scalar $\lambda$.

Lemma 3.3. Suppose $r$ and $s$ are nonnegative integers with $(r, s) \neq(0,0)$. Let $X$ be a complex Banach space. If $A, A^{\prime} \in \mathcal{B}(X)$ satisfy $\sigma_{\pi}\left(B^{r} A B^{s}+B^{s} A B^{r}\right)=\sigma_{\pi}\left(B^{r} A^{\prime} B^{s}+\right.$ $\left.B^{s} A^{\prime} B^{r}\right)$ for all $B \in \mathcal{I}_{1}(X)$, then $A=A^{\prime}$.

Proof. We may suppose that $A^{\prime} \neq 0$ since it is obvious that $\sigma_{\pi}\left(B^{r} A B^{s}+B^{s} A B^{r}\right)=\{0\}$ for all rank one idempotents $B$ implies that $A=0$.

Assume first that $s \geq r>0$. Then the assumption implies that $\sigma_{\pi}(B A B)=\sigma_{\pi}\left(B A^{\prime} B\right)$ and hence $f(A x)=\operatorname{tr}(B A B)=\operatorname{tr}\left(B A^{\prime} B\right)=f\left(A^{\prime} x\right)$ for all rank one idempotents $B=x \otimes f$. By Lemma 3.2, we see that $A^{\prime}=A$.

Assume then that $s>r=0$ and write the rank-one idempotent $B$ in the form $B=x \otimes f$ with $\langle x, f\rangle=1$. Then $A B^{s}+B^{s} A=A B+B A$, and $\operatorname{tr}(A B+B A)=0$ if and only if $\sigma_{\pi}(A B+B A)=\{0\}$ or $\{\beta,-\beta\}$ for some nonzero $\beta$. Since $\sigma_{\pi}(A B+B A)=\sigma_{\pi}\left(A^{\prime} B+B A^{\prime}\right)$, we see that $\operatorname{tr}(A B+B A)=0$ if and only if $\operatorname{tr}\left(A^{\prime} B+B A^{\prime}\right)=0$. It follows from Lemma 3.2 again that $A^{\prime}=\lambda A$ for some scalar $\lambda$. But the peripheral spectrum coincidence implies $\lambda=1$.

As a direct consequence of Lemma 3.3 and the condition (3.1), we have

Corollary 3.4. Let $\Phi$ satisfy the hypothesis of Theorem 3.1. Then $\Phi$ is injective, and $\Phi(0)=0$.

To complete the proof of Theorem 3.1, we need some more technical lemmas.

Lemma 3.5. Let $P, Q \in \mathcal{I}_{1}(X)$. Then $P Q=0=Q P$ if and only if there is $B \in \mathcal{B}(X)$, which can be chosen to have rank 2, such that $\sigma_{\pi}(P B+B P)=\{2\}, \sigma_{\pi}(Q B+B Q)=\{-2\}$, and $\sigma_{\pi}(B R+R B)=\{0\}$ whenever $R \in \mathcal{I}_{1}(X)$ satisfies $\sigma_{\pi}(P R+R P)=\sigma_{\pi}(Q R+R Q)=\{0\}$. 
Proof. Suppose $P, Q \in \mathcal{I}_{1}(X)$ satisfy $P Q=0=Q P$. Then there is a space decomposition for $X$ such that $P$ and $Q$ have operator matrices

$$
\left(\begin{array}{ll}
1 & 0 \\
0 & 0
\end{array}\right) \oplus 0 \text { and } \quad\left(\begin{array}{cc}
0 & 0 \\
0 & 1
\end{array}\right) \oplus 0
$$

Using the same space decomposition, let $B$ have operator matrix $\left(\begin{array}{cc}1 & 0 \\ 0 & -1\end{array}\right) \oplus 0$. Then $B$ has rank 2 such that $\sigma_{\pi}(P B+B P)=\{2\}$ and $\sigma_{\pi}(Q B+B Q)=\{-2\}$. Consider any $R \in \mathcal{I}_{1}(X)$ such that $\sigma_{\pi}(P R+R P)=\sigma_{\pi}(Q R+R Q)=\{0\}$. Using the same space decomposition as $P$ and $Q$, we assume that $R$ has operator matrix

$$
\left(\begin{array}{ll}
R_{11} & R_{12} \\
R_{21} & R_{22}
\end{array}\right)
$$

where $R_{11} \in M_{2}$. Since $\sigma_{\pi}(P R+R P)=\sigma_{\pi}(Q R+R Q)=\{0\}$, the $(1,1)$ and $(2,2)$ entry of $R_{11}$ are both zero. Thus, $R_{22}$ has trace one and rank one. We may then assume that $R_{22}$ has operator matrix $(1) \oplus 0$. As a result, we may assume that the operator matrix of $R$ has the form $\hat{R} \oplus 0$, where $R$ or $R^{t}$ has one of the following forms:

$$
\left(\begin{array}{lll}
0 & 0 & * \\
0 & 0 & * \\
0 & 0 & 1
\end{array}\right) \quad \text { or } \quad\left(\begin{array}{lll}
0 & a & b \\
0 & 0 & 0 \\
0 & c & 1
\end{array}\right) \quad \text { with } a=b c
$$

Consequently, $\sigma_{\pi}(B R+R B)=\{0\}$.

Conversely, suppose $P, Q \in \mathcal{I}_{1}(X)$ such that $P Q \neq 0$ or $Q P \neq 0$. Then there is a space decomposition for $X=X_{1} \oplus X_{2}$ with $\operatorname{dim} X_{1}=2 \operatorname{such}$ that $P$ has operator matrix $\left(\begin{array}{cc}1 & 0 \\ 0 & 0\end{array}\right) \oplus$ 0 and $Q$ has operator matrix

$$
\left(\begin{array}{ll}
0 & 0 \\
1 & 1
\end{array}\right) \oplus 0 \quad \text { or } \quad\left(\begin{array}{ll}
0 & 1 \\
0 & 1
\end{array}\right) \oplus 0
$$

We assume that the former case holds. The proof for the other case is similar. Suppose there is a $B$ in $\mathcal{B}(X)$ such that $\sigma_{\pi}(P B+B P)=\{2\}, \sigma_{\pi}(Q B+B Q)=\{-2\}$ and $\sigma_{\pi}(B R+R B)=\{0\}$ whenever $R$ in $\mathcal{I}_{1}(X)$ satisfies $\sigma_{\pi}(P R+R P)=\sigma_{\pi}(Q R+R Q)=\{0\}$. Using the same space decomposition as $P$ and $Q$, we assume that $B$ has operator matrix

$$
\left(\begin{array}{ll}
B_{11} & B_{12} \\
B_{21} & B_{22}
\end{array}\right)
$$

where $B_{11} \in M_{2}$. 
First, we claim that $B_{22}=0$. If not, we may assume that the $(1,1)$ entry of $B_{22}$ is nonzero. If $R$ has operator matrix

$$
\left(\begin{array}{lll}
0 & 0 & 0 \\
0 & 0 & 0 \\
0 & 0 & 1
\end{array}\right) \oplus 0
$$

we see that $\sigma_{\pi}(P R+R P)=\sigma_{\pi}(Q R+R Q)=\{0\} \neq \sigma_{\pi}(B R+R B)$.

Next, we claim that $B_{12}=0$. If this is not true, we can find a suitable space decomposition for $X_{2}$ such that $B_{12}$ has the form $\left(\begin{array}{ll}1 & 0 \\ 0 & T\end{array}\right)$, where the last column is vacuous if $\operatorname{dim} X=3$, and $T$ has rank zero or one. But then if $R \in \mathcal{I}_{1}(X)$ has operator matrix

$$
\left(\begin{array}{lll}
0 & 0 & 0 \\
0 & 0 & 0 \\
1 & 0 & 1
\end{array}\right) \oplus 0
$$

we have $\sigma_{\pi}(B R+R B) \neq\{0\}$. Similarly, we can show that $B_{21}=0$.

$$
\text { Now, we consider } B_{11}=\left(\begin{array}{cc}
b_{11} & b_{12} \\
b_{21} & b_{22}
\end{array}\right) \text {. Let } R \text { has operator matrix }\left(\begin{array}{ccc}
0 & 0 & 0 \\
1 & 0 & 1 \\
1 & 0 & 1
\end{array}\right) \oplus 0 \text {, We }
$$

see that $\sigma_{\pi}(P R+R P)=\sigma_{\pi}(Q R+R Q)=\{0\}$. Because $B R+R B$ has operator matrix

$$
\left(\begin{array}{ccc}
b_{12} & 0 & b_{12} \\
b_{11}+b_{22} & b_{12} & b_{22} \\
b_{11} & b_{12} & 0
\end{array}\right) \oplus 0
$$

so $b_{12}=0$. Since $\sigma_{\pi}(P B+B P)=\{2\}$ and $\sigma_{\pi}(Q B+Q B)=\{-2\}$, it follows that $b_{11}=$ $1, b_{22}=-1$. Finally, for $R$ with operator matrix $\left(\begin{array}{cc}0 & -1 \\ 0 & 1\end{array}\right) \oplus 0$, we have $\sigma_{\pi}(P R+R P)=$ $\sigma_{\pi}(Q R+R Q)=\{0\}$. But $B R+R B$ has operator matrix

$$
\left(\begin{array}{cc}
-b_{21} & 0 \\
b_{21} & -2-b_{21}
\end{array}\right) \oplus 0
$$

which cannot be a nilpotent.

For a Banach space $X$ and a ring automorphism $\tau$ of $\mathbb{C}$, if an additive map $T: X \rightarrow X$ satisfies $T(\lambda x)=\tau(\lambda) T x$ for all complex $\lambda$ and all vectors $x$, we say that $T$ is $\tau$-linear.

The following result can be proved by a similar argument to the proof of the main result in [15], see also [3] and [21].

Lemma 3.6. Let $X_{1}$ and $X_{2}$ be complex Banach spaces with dimension at least 3. Let $\Phi: \mathcal{I}_{1}\left(X_{1}\right) \rightarrow \mathcal{I}_{1}\left(X_{2}\right)$ be a bijective map with the property that $P Q=Q P=0 \Leftrightarrow \Phi(P) \Phi(Q)=$ 
$\Phi(Q) \Phi(P)=0$ for all $P, Q \in \mathcal{I}_{1}\left(X_{1}\right)$. Then there exists a ring automorphism $\tau$ of $\mathbb{C}$ such that one of the following cases holds.

(i) There exists a $\tau$-linear transformation $T: X_{1} \rightarrow X_{2}$ satisfying $\Phi(P)=T P T^{-1}$ for all $P \in \mathcal{I}_{1}\left(X_{1}\right)$.

(ii) There exists a $\tau$-linear transformation $T: X_{1}^{*} \rightarrow X_{2}$ satisfying $\Phi(P)=T P^{*} T^{-1}$ for all $P \in \mathcal{I}_{1}\left(X_{1}\right)$.

If $X$ is infinite dimensional, the transformation $T$ is an invertible bounded linear or conjugate linear operator.

In the following, we present the proof of Theorem 3.1.

Proof of Theorem 3.1. Recall that $\Phi$ satisfies condition (3.1).

Case 1. $s>r>0$.

Claim 1.1. $\Phi$ is injective, and $\Phi(0)=0$.

It is just Corollary 3.4.

Claim 1.2. $\Phi$ preserves rank one operators in both directions.

Assume that $\operatorname{rank} A=1$; then Claim 1.1 implies that $\Phi(A) \neq 0$. For any $B \in \mathcal{A}_{1}$, by Lemma 2.1, $\sigma_{\pi}\left(\Phi(B)^{r} \Phi(A) \Phi(B)^{s}+\Phi(B)^{s} \Phi(A) \Phi(B)^{r}\right)=\sigma_{\pi}\left(B^{r} A B^{s}+B^{s} A B^{r}\right)$ has at most two different elements. Since the range of $\Phi$ contains all operators of rank at most three, for any $C \in \mathcal{A}_{2}$ with $\left.\operatorname{rank}(C) \leq 3, \sigma_{\pi}\left(C^{r} \Phi(A) C^{s}\right)+C^{s} \Phi(A) C^{r}\right)$ has at most two different elements. Applying Lemma 2.1 again one sees that $\Phi(A)$ is of rank one. Conversely, assume that $T \in \mathcal{A}_{2}$ is of rank one. Then, there is $A \in \mathcal{A}_{1}$ such that $\Phi(A)=T$. For any $B \in \mathcal{A}_{1}$, Lemma 2.1 implies that $\left.\sigma_{\pi}\left(B^{r} A B^{s}+B^{s} A B^{r}\right)=\sigma_{\pi}\left(\Phi(B)^{r} \Phi(A) \Phi(B)^{s}\right)+\Phi(B)^{s} \Phi(A) \Phi(B)^{r}\right)$ has at most two different elements. Applying Lemma 2.1 again one gets $A$ is of rank one.

Claim 1.3. $\Phi$ is linear.

We show first that $\Phi$ is additive.

Observe that, for any operator $A$ and rank one operator $x \otimes f$, we have

$$
(x \otimes f)^{r} A(x \otimes f)^{s}+(x \otimes f)^{s} A(x \otimes f)^{r}=2\langle x, f\rangle^{s+r-2}(x \otimes f) A(x \otimes f),
$$

and hence

$$
\begin{aligned}
& \sigma_{\pi}\left((x \otimes f)^{r} A(x \otimes f)^{s}+(x \otimes f)^{s} A(x \otimes f)^{r}\right) \\
= & \operatorname{Tr}\left(2\langle x, f\rangle^{s+r-2}(x \otimes f) A(x \otimes f)\right)=\left\{2\langle x, f\rangle^{s+r-1}\langle A x, f\rangle\right\} .
\end{aligned}
$$

Let $A, B \in \mathcal{A}_{1}$ be arbitrary. For any $y \in X_{2}, g \in X_{2}^{*}$ with $\langle y, g\rangle=1$, Claim 2 implies that there exist $x \in X_{1}, f \in X_{1}^{*}$ such that $\Phi(x \otimes f)=y \otimes g$. 
Then, by Eqs.(3.1) and (3.3), we have

$$
\begin{aligned}
\{2\langle\Phi(A+B) y, g\rangle\langle y, g\rangle\}= & \sigma_{\pi}\left((y \otimes g)^{r} \Phi(A+B)(y \otimes g)^{s}+(y \otimes g)^{s} \Phi(A+B)(y \otimes g)^{r}\right) \\
= & \sigma_{\pi}\left((x \otimes f)^{r}(A+B)(x \otimes f)^{s}+(x \otimes f)^{s}(A+B)(x \otimes f)^{r}\right) \\
= & \left\{\operatorname{Tr}\left(2\langle x, f\rangle^{r+s-2}(x \otimes f)(A+B)(x \otimes f)\right)\right\} \\
= & \left\{\operatorname{Tr}\left(2\langle x, f\rangle^{r+s-2}(x \otimes f) A(x \otimes f)\right)\right\} \\
& +\left\{\operatorname{Tr}\left(2\langle x, f\rangle^{r+s-2}(x \otimes f) B(x \otimes f)\right)\right\} \\
= & \left\{\operatorname{Tr}\left(2\langle y, g\rangle^{s+r-2}(y \otimes g) \Phi(A)(y \otimes g)\right)\right. \\
& +\left\{\operatorname{Tr}\left(2\langle y, g\rangle^{s+r-2}(y \otimes g) \Phi(B)(y \otimes g)\right)\right. \\
= & \left\{\operatorname{Tr}\left(2\langle y, g\rangle^{s+r-2}(y \otimes g)(\Phi(A)+\Phi(B))(y \otimes g)\right)\right. \\
= & \{\langle(\Phi(A)+\Phi(B)) y, g\rangle\langle y, g\rangle\} .
\end{aligned}
$$

It follows that

$$
\langle\Phi(A+B) y, g\rangle=\langle(\Phi(A)+\Phi(B)) y, g\rangle
$$

holds for any $y \in X_{2}, g \in X_{2}^{*}$ with $\langle y, g\rangle=1$. This entails $\Phi(A+B)=\Phi(A)+\Phi(B)$ and hence $\Phi$ is additive. Similarly one can check that $\Phi$ is homogeneous, I.e., $\Phi(\lambda A)=\lambda \Phi(A)$. So $\Phi$ is linear.

The claims 1.1-1.3 imply that $\Phi$ is an injective linear map preserving rank one operators in both directions. By [5] the following claim is true.

Claim 1.4. One of the following statements holds:

(i) There exist two linear bijections $T: X_{1} \rightarrow X_{2}$ and $S: X_{1}^{*} \rightarrow X_{2}^{*}$ such that $\Phi(x \otimes f)=$ $T x \otimes S f$ for all rank one operators $x \otimes f \in \mathcal{A}_{1}$.

(ii) There exist two linear bijections $T: X_{1}^{*} \rightarrow X_{2}$ and $S: X_{1} \rightarrow X_{2}^{*}$ such that $\Phi(x \otimes f)=$ $T f \otimes S x$ for all rank one operators $x \otimes f \in \mathcal{A}_{1}$.

Claim 1.5. There exists a scalar $\lambda \in \mathbb{C}$ with $\lambda^{m}=1$ and $m=r+s+1$ such that, if (i) occurs in Claim 1.4, then $\langle T x, S f\rangle=\lambda\langle x, f\rangle$ holds for all $x \in X_{1}$ and $f \in X_{1}^{*}$; if (ii) occurs in Claim 4, then $\langle T f, S x\rangle=\lambda\langle x, f\rangle$ holds for all $x \in X_{1}$ and $f \in X_{1}^{*}$.

To check Claim 1.5, we first assume that the case (i) in Claim 1.4 occurs. Then, for any $x \in X_{1}, f \in X_{1}^{*}$, we have $\sigma_{\pi}\left(2(x \otimes f)^{m}\right)=\left\{2\langle x, f\rangle^{m}\right\}=\sigma_{\pi}\left(2(T x \otimes S f)^{m}\right)=\left\{2\langle T x, S f\rangle^{m}\right\}$. So $\langle T x, S f\rangle=\lambda_{x, f}\langle x, f\rangle$ with $\lambda_{x, f}^{m}=1$. Especially, $\langle x, f\rangle=0 \Leftrightarrow\langle T x, S f\rangle=0$.

Let $V_{0}=\{(x, f) \mid\langle x, f\rangle=0\}, V_{t}=\left\{(x, f) \mid \lambda_{x, f}=e^{i \frac{2(t-1) \pi}{m}}\right\}, t=1, \ldots, m$. Then $\bigcup_{t=1}^{m} V_{t}=X \times X^{*}$ and $V_{k} \cap V_{j}=V_{0}$ if $k \neq j, k, j=1,2, \ldots, m$. For $x_{1}, x_{2} \in X_{1}$ we denote by $\left[x_{1}, x_{2}\right]$ the linear subspace spanned by $x_{1}$ and $x_{2}$.

Assertion 1. For any nonzero $x_{1}, x_{2} \in X_{1}, f \in X_{1}^{*}$, there exists some $k \in\{1,2, \ldots, m\}$ such that $\left[x_{1}, x_{2}\right] \times[f] \subseteq V_{k}$. 
We need only to show that we may take $\lambda_{x_{1}, f}$ and $\lambda_{x_{2}, f}$ such that $\lambda_{x_{1}, f}=\lambda_{x_{2}, f}$. Consider the following three cases.

Case $\mathbf{1}^{\circ} \cdot x_{1}, x_{2}$ are linearly dependent.

Assume that $x_{2}=\alpha x_{1}$; then $\alpha \neq 0$ and $\alpha \lambda_{x_{1}, f}\left\langle x_{1}, f\right\rangle=\alpha\left\langle T x_{1}, S f\right\rangle=\left\langle T x_{2}, S f\right\rangle=$ $\alpha \lambda_{x_{2}, f}\left\langle x_{1}, f\right\rangle$. So we may take $\lambda_{x_{1}, f}$ and $\lambda_{x_{2}, f}$ such that $\lambda_{x_{1}, f}=\lambda_{x_{2}, f}$.

Case $\mathbf{2}^{\circ} \cdot x_{1}, x_{2}$ are linearly independent and at least one of $\left\langle x_{i}, f\right\rangle, i=1,2$ is not zero.

In this case, for any $\alpha, \beta \in \mathbb{C}$ we have

$$
\alpha \lambda_{\alpha, \beta}\left\langle x_{1}, f\right\rangle+\beta \lambda_{\alpha, \beta}\left\langle x_{2}, f\right\rangle=\left\langle T\left(\alpha x_{1}+\beta x_{2}\right), S f\right\rangle=\alpha \lambda_{x_{1}, f}\left\langle x_{1}, f\right\rangle+\beta \lambda_{x_{2}, f}\left\langle x_{2}, f\right\rangle,
$$

where $\lambda_{\alpha, \beta}=\lambda_{\alpha x_{1}+\beta x_{2}, f}$. Let

$$
\eta=\left(\begin{array}{c}
\lambda_{x_{1}, f}\left\langle x_{1}, f\right\rangle \\
\lambda_{x_{2}, f}\left\langle x_{2}, f\right\rangle
\end{array}\right), \eta_{0}=\left(\begin{array}{c}
\left\langle x_{1}, f\right\rangle \\
\left\langle x_{2}, f\right\rangle
\end{array}\right), \xi=\left(\begin{array}{c}
\alpha \\
\beta
\end{array}\right) \in \mathbb{C}^{2} .
$$

Then Eq.(3.2) implies that

$$
\langle\eta, \xi\rangle=\lambda_{\alpha, \beta}\left\langle\eta_{0}, \xi\right\rangle
$$

holds for any $\xi \in \mathbb{C}^{2}$. It follows that $\langle\eta, \xi\rangle=0 \Leftrightarrow\left\langle\eta_{0}, \xi\right\rangle=0$. So, as the vectors in $\mathbb{C}^{2}$, we must have $\eta=\gamma \eta_{0}$ for some scalar $\gamma$. Now it is clear that $\lambda_{x_{1}, f}=\lambda_{x_{2}, f}$.

Case $3^{\circ} \cdot x_{1}, x_{2}$ are linearly independent and $\left\langle x_{1}, f\right\rangle=\left\langle x_{2}, f\right\rangle=0$.

Then $\left\langle T x_{1}, S f\right\rangle=\lambda_{x_{1}, f}\left\langle x_{1}, f\right\rangle=0=\left\langle T x_{2}, S f\right\rangle=\lambda_{x_{2}, f}\left\langle x_{2}, f\right\rangle$. In this case it is clear that we can take $\lambda_{x_{1}, f}$ and $\lambda_{x_{2}, f}$ such that $\lambda_{x_{1}, f}=\lambda_{x_{2}, f}$.

Similar to the previous discussion, we have

Assertion 2. For any nonzero $x \in X_{1}, f_{1}, f_{2} \in X_{1}^{*}$, there exists some $k \in\{1,2, \ldots, m\}$ such that $[x] \times\left[f_{1}, f_{2}\right] \subseteq V_{k}$.

Assertion 3. There exists a scalar $\lambda \in \mathbb{C}$ with $\lambda^{m}=1$ such that $\lambda_{x, f}=\lambda$ for all $x \in X_{1}$ and $f \in X_{1}^{*}$.

For any $f_{0} \neq 0$, there exist $x_{0}$ such that $\left\langle x_{0}, f_{0}\right\rangle=1$. Then $\left\langle T x_{0}, S f_{0}\right\rangle=\lambda_{x_{0}, f_{0}}$ and $\left(x_{0}, f_{0}\right) \in V_{k_{0}}$ for some $k_{0} \in\{1,2, \ldots, m\}$. So, by Assertion 1 , for any $x \in X_{1}$, we have $\left[x, x_{0}\right] \times\left[f_{0}\right] \subseteq V_{k_{0}}$, which implies that $X_{1} \times\left[f_{0}\right] \subseteq V_{k_{0}}$. Similarly, by Assertion 2 one gets, for any $x_{0} \neq 0,\left[x_{0}\right] \times X_{1}^{*} \subseteq V_{k_{0}}$. Thus we obtain that $X_{1} \times X_{1}^{*}=V_{k_{0}}$.

Hence, there exists a scalar $\lambda \in \mathbb{C}$ with $\lambda^{m}=1$ such that $\lambda_{x, f}=\lambda$ for all $x$ and $f$, that is, $\langle T x, S f\rangle=\lambda\langle x, f\rangle$ holds for all $x \in X_{1}$ and $f \in X_{1}^{*}$. So Assertion 3 is true.

This completes the proof of Claim 1.5 for the case (i) of Claim 1.4.

If the case (ii) in Claim 1.4 occurs, by a similar argument one can show that there exists a scalar $\lambda$ with $\lambda^{m}=1$ such that $\langle T f, S x\rangle=\lambda\langle x, f\rangle$ holds for all $x \in X_{1}$ and $f \in X_{1}^{*}$. Hence the last conclusion of Claim 1.5 is also true. 
Claim 1.6. There exists a scalar $\lambda$ with $\lambda^{m}=1$ such that one of the followings holds:

(1) There exists an invertible operator $T \in \mathcal{B}\left(X_{1}, X_{2}\right)$ such that $\Phi(x \otimes f)=\lambda T(x \otimes f) T^{-1}$ for all $x \otimes f \in \mathcal{A}_{1}$.

(2) $X_{1}$ and $X_{2}$ are reflexive, and there exists an invertible operator $T \in \mathcal{B}\left(X_{1}^{*}, X_{2}\right)$ such that $\Phi(x \otimes f)=\lambda T(x \otimes f)^{*} T^{-1}$ for all $x \otimes f \in \mathcal{A}_{1}$.

Suppose that the case (i) of Claim 1.4 occurs. Then by Claim 1.5, there exists a scalar $\lambda \in \mathbb{C}$ with $\lambda^{m}=1$ such that $\langle T x, S f\rangle=\lambda\langle x, f\rangle$ holds for all $x \in X_{1}$ and $f \in X_{1}^{*}$. If $\left\{x_{n}\right\} \subset X_{1}$ is a sequence such that $x_{n} \rightarrow x$ and $T x_{n} \rightarrow y$ for some $x \in X_{1}$ and $y \in X_{2}$ as $n \rightarrow \infty$, then, for any $f \in X_{1}^{*}$, we have $\langle y, S f\rangle=\lim _{n \rightarrow \infty}\left\langle T x_{n}, S f\right\rangle=\lim _{n \rightarrow \infty} \lambda\left\langle x_{n}, f\right\rangle=\lambda\langle x, f\rangle=\langle T x, S f\rangle$. As $S$ is surjective we must have $y=T x$. So the bijection $T$ is a closed operator and thus a bounded invertible operator. Since $\langle T x, S f\rangle=\left\langle x, T^{*} S f\right\rangle=\lambda\langle x, f\rangle$ holds for all $x \in X_{1}$ and $f \in X_{1}^{*}$, we see that $T^{*} S=\lambda I$, that is $S=\lambda\left(T^{*}\right)^{-1}$. It follows from the case (i) of Claim 1.4 that $\Phi(x \otimes f)=T x \otimes S f=\lambda T x \otimes\left(T^{*}\right)^{-1} f=\lambda T(x \otimes f) T^{-1}$ for any rank one operator $x \otimes f$, i.e., the case (1) of Claim 1.6 holds.

Suppose that the case (ii) of Claim 1.4 occurs. Then by Claim 1.5, there exists a scalar $\lambda \in \mathbb{C}$ with $\lambda^{m}=1$ such that $\langle T f, S x\rangle=\lambda\langle x, f\rangle$ holds for all $x \in X_{1}$ and $f \in X_{1}^{*}$. Similar to the above argument we can check that both $T$ and $S$ are bounded invertible operators with $S=\lambda\left(T^{*}\right)^{-1}$. It follows that $\Phi(x \otimes f)=\lambda T(x \otimes f)^{*} T^{-1}$ for any $x \otimes f$, obtaining that the case (2) of Claim 1.6 holds. Moreover, by [5], in this case both $X_{1}$ and $X_{2}$ are reflexive.

Claim 1.7. The theorem is true.

Assume that we have the case (1) of Claim 1.6. Let $A \in \mathcal{A}_{1}$ be arbitrary. For any $x \in X_{1}$ and $f \in X_{1}^{*}$ with $\langle x, f\rangle=1$, we have

$$
\begin{aligned}
\{2\langle A x, f\rangle\}= & \sigma_{\pi}\left((x \otimes f)^{r} A(x \otimes f)^{s}+(x \otimes f)^{s} A(x \otimes f)^{r}\right) \\
= & \sigma_{\pi}\left(\left(\lambda T(x \otimes f) T^{-1}\right)^{r} \Phi(A)\left(\lambda T(x \otimes f) T^{-1}\right)^{s}\right. \\
& \left.+\left(\lambda T(x \otimes f) T^{-1}\right)^{s} \Phi(A)\left(\lambda T(x \otimes f) T^{-1}\right)^{r}\right) \\
= & \sigma_{\pi}\left(\frac{2}{\lambda}(x \otimes f) T^{-1} \Phi(A) T(x \otimes f)\right) \\
= & \left\{\left\langle\frac{2}{\lambda} T^{-1} \Phi(A) T x, f\right\rangle\right\} .
\end{aligned}
$$

This implies that $\Phi(A)=\lambda T A T^{-1}$ for any $A \in \mathcal{A}_{1}$.

A similar argument shows that if the case (2) of Claim 1.6 occurs then $\Phi$ has the form given in (2) of Theorem 1.2.

Case 2. $s>r=0$.

Claim 2.1. $\Phi$ is injective, and $\Phi(0)=0$.

It is just Corollary 3.4. 
Claim 2.2. If $A \in \mathcal{A}_{1}$ is a nonzero multiple of a rank one idempotent, then so is $\Phi(A)$. In particular, if $P \in \mathcal{I}_{1}\left(X_{1}\right)$, then $\Phi(P)=\mu R$ such that $R \in \mathcal{I}_{1}\left(X_{2}\right)$ and $\mu^{s+1}=1$.

Let $A \neq 0$ be a nonzero multiple of an idempotent, say $A=\alpha P$, where $0 \neq \alpha \in \mathbb{C}$ and $P \in \mathcal{I}_{1}\left(X_{1}\right)$. For any $D$ in $\mathcal{A}_{2}$ of rank at most 3 , there is $C$ in $\mathcal{A}_{1}$ such that $\Phi(C)=D$. By equation (3.1) we have $\sigma_{\pi}\left(\Phi(A) D^{s}+D^{s} \Phi(A)\right)=\sigma_{\pi}\left(A C^{s}+C^{s} A\right)$, which contains at most two different elements. Putting $B=A$ in equation (3.1), we have $\sigma_{\pi}\left(2 \Phi(A)^{s+1}\right)=\sigma_{\pi}\left(2 A^{s+1}\right) \neq$ $\{0\}$. Applying Corollary 2.3, depending on $s>r=0$, we see that $\Phi(A)$ is a nonzero multiple of rank one idempotent. Thus $\Phi$ preserves nonzero multiples of rank one idempotents. If $P$ in $\mathcal{A}_{1}$ is a rank one idempotent, then $\Phi(P)=\mu R$, where $R \in \mathcal{I}_{1}\left(X_{2}\right)$ and $\mu \in \mathbb{C}$. Since $\{2\}=\sigma_{\pi}\left(2 P^{s+1}\right)=\sigma_{\pi}\left(2 \Phi(P)^{s+1}\right)=\left\{2 \mu^{s+1}\right\}$, we see that $\mu^{s+1}=1$.

Claim 2.3. There exists a scalar $\lambda$ with $\lambda^{s+1}=1$ such that $\lambda^{-1} \Phi$ sends rank one idempotents to rank one idempotents.

Let $0 \neq f \in X_{1}^{*}$. Assume that $\left\langle x_{1}, f\right\rangle=\left\langle x_{2}, f\right\rangle=1$. By Claim 2, $\Phi\left(x_{1} \otimes f\right)=\lambda_{1} y_{1} \otimes g_{1}$ and $\Phi\left(x_{2} \otimes f\right)=\lambda_{2} y_{2} \otimes g_{2}$, where $g_{1}\left(y_{1}\right)=g_{2}\left(y_{2}\right)=1$ and $\lambda_{1}^{s+1}=\lambda_{2}^{s+1}=1$. Using the peripheral spectrum equation (3.1) we have

$$
\begin{aligned}
& \sigma_{\pi}\left(\lambda_{1}^{s} \lambda_{2}\left(\left(y_{1} \otimes g_{1}\right)\left(y_{2} \otimes g_{2}\right)+\left(y_{2} \otimes g_{2}\right)\left(y_{1} \otimes g_{1}\right)\right)\right. \\
= & \sigma_{\pi}\left(\lambda_{1} \lambda_{2}^{s}\left(y_{1} \otimes g_{1}\right)\left(y_{2} \otimes g_{2}\right)+\left(y_{2} \otimes g_{2}\right)\left(y_{1} \otimes g_{1}\right)\right) \\
= & \sigma_{\pi}\left(\left(x_{1} \otimes f\right)\left(x_{2} \otimes f\right)+\left(x_{2} \otimes f\right)\left(x_{1} \otimes f\right)\right) \\
= & \{2\} .
\end{aligned}
$$

Then $\lambda_{1}^{s} \lambda_{2}=\lambda_{2}^{s} \lambda_{1}$. In particular, $\lambda_{1}^{2}=\lambda_{2}^{2}$ as $\lambda_{1}^{s+1}=1=\lambda_{2}^{s+1}$. Suppose $\lambda_{1}=-\lambda_{2}$, then we have $\sigma_{\pi}\left(\left(y_{1} \otimes g_{1}\right)\left(y_{2} \otimes g_{2}\right)+\left(y_{2} \otimes g_{2}\right)\left(y_{1} \otimes g_{1}\right)\right)=\{-2\}$, but by Lemma 2.4, this is impossible. So, $\lambda_{1}=\lambda_{2}$. Denote this common value by $\lambda_{f}$. Similarly, for any nonzero $x$ in $X_{1}$ we will have an $m$ th root $(m=s+1) \lambda_{x}$ of unity depending only on $x$ such that $\Phi(x \otimes f)=\lambda_{x} Q_{x \otimes f}$, for some rank one idempotent $Q_{x \otimes f}$ whenever $f(x)=1$.

Now consider any two rank one idempotents $x_{1} \otimes f_{1}$ and $x_{2} \otimes f_{2}$ in $\mathcal{A}_{1}$. We write $x_{1} \otimes f_{1} \sim$ $x_{2} \otimes f_{2}$ if there is a scalar $\lambda$ with $\lambda^{s+1}=1$ such that $\lambda \Phi\left(x_{i} \otimes f_{i}\right)$ is a rank one idempotent for $i=1,2$. In case $\alpha=\left\langle x_{1}, f_{2}\right\rangle \neq 0$, we see that

$$
x_{1} \otimes f_{1} \sim x_{1} \otimes \frac{f_{2}}{\alpha}=\frac{x_{1}}{\alpha} \otimes f_{2} \sim x_{2} \otimes f_{2} .
$$

In case $\left\langle x_{1}, f_{2}\right\rangle=\left\langle x_{2}, f_{1}\right\rangle=0$, we also have

$$
x_{1} \otimes f_{1} \sim\left(x_{1}+x_{2}\right) \otimes f_{1} \sim\left(x_{1}+x_{2}\right) \otimes f_{2} \sim x_{2} \otimes f_{2}
$$

So, Claim 2.3 is true.

By Claim 2.3, without loss of generality, we assume that $\Phi$ preserves rank one idempotents. 
Claim 2.4. If $\Phi(A) \in \mathcal{A}_{2}$ is a rank one idempotent, then $A \in \mathcal{A}_{1}$ is a rank one idempotent.

Assume that $\Phi(A)$ is a rank one idempotent. Suppose $A$ is not a rank one idempotent, i.e., $A$ is rank one nilpotent or $A$ has rank at least two. Putting $B=A$ in equation (3.1), we have $\sigma_{\pi}\left(2 A^{s+1}\right)=\sigma_{\pi}\left(2 \Phi(A)^{s+1}\right)=\{2\}$, so $A^{2} \neq 0$. Then $A$ has rank at least 2 . In this case, by the arguments in the proof of Lemma 2.2, for such $A$ we can find a operator $B$ with $\operatorname{rank} B \leq 3$ such that $\sigma_{\pi}\left(A B^{s}+B^{s} A\right)$ has three different points. But $\sigma_{\pi}\left(\Phi(A) \Phi(B)^{s}+\Phi(B)^{s} \Phi(A)\right)$ has at most two different points, a contradiction.

Claim 2.5. One of the following statements is true.

(i) There exists a bounded invertible linear operator $T: X_{1} \rightarrow X_{2}$ such that

$$
\Phi(x \otimes f)=T(x \otimes f) T^{-1} \quad \text { for all } \quad x \in X_{1}, f \in X_{1}^{*} \text { with }\langle\mathrm{x}, \mathrm{f}\rangle=1
$$

(ii) There exists a bounded invertible linear operator $T: X_{1}^{*} \rightarrow X_{2}$ such that

$$
\Phi(x \otimes f)=T(x \otimes f)^{*} T^{-1} \quad \text { for all } \quad x \in X_{1}, f \in X_{1}^{*} \text { with }\langle\mathrm{x}, \mathrm{f}\rangle=1 .
$$

Since $\Phi$ preserves rank one idempotents in both directions, by use of Lemma 3.5, it is easily checked that $P, Q \in \mathcal{I}_{1}\left(X_{1}\right)$ satisfy $P Q=0=Q P$ if and only if $\Phi(P) \Phi(Q)=0=\Phi(Q) \Phi(P)$. Thus we can apply Lemma 3.6 to conclude that (i) or (ii) holds, but with $T$ a $\tau$-linear for some ring automorphism $\tau$ of $\mathbb{C}$.

Next we prove that $\tau$ is the identity and hence $T$ is linear. For any $\alpha \in \mathbb{C} \backslash\{0,1\}$, let $A$ and $B$ have respectively operator matrices

$$
\left(\begin{array}{cc}
1 & \alpha-1 \\
0 & 0
\end{array}\right) \oplus 0 \quad \text { and } \quad\left(\begin{array}{cc}
1 & 0 \\
1 & 0
\end{array}\right) \oplus 0 .
$$

Then

$$
A B^{s}+B^{s} A=\left(\begin{array}{cc}
\alpha+1 & \alpha-1 \\
1 & \alpha-1
\end{array}\right) \oplus 0 .
$$

Since

$$
\begin{gathered}
\sigma_{\pi}\left(A B^{s}+B^{s} A\right)=\sigma_{\pi}\left(\Phi(A) \Phi(B)^{s}+\Phi(B)^{s} \Phi(A)\right) \\
=\sigma_{\pi}\left(T\left(A B^{s}+B^{s} A\right) T^{-1}\right)=\left\{\tau(\xi): \xi \in \sigma_{\pi}(A B+B A)\right\},
\end{gathered}
$$

Note that $\sigma_{\pi}\left(A B^{s}+B^{s} A\right)=\{\alpha \pm \sqrt{\alpha}\}=\sigma_{\pi}(\tau(\alpha \pm \sqrt{\alpha}))$. It follows that $\tau(2 \alpha)=\tau(\alpha+$ $\sqrt{\alpha}+\alpha-\sqrt{\alpha})=\alpha+\sqrt{\alpha}+\alpha-\sqrt{\alpha}=2 \alpha$. Hence $\tau(\alpha)=\alpha$ for any $\alpha \in \mathbb{C}$. It follows that $T$ is an invertible bounded linear operator.

Claim 2.6. $\Phi$ has the form in Theorem 3.1. 
Suppose (i) in Claim 2.5 holds. Let $A \in \mathcal{A}_{1}$ be arbitrary. For any $x \in X_{1}$ and $f \in X_{1}^{*}$ with $\langle x, f\rangle=1$, the condition (3.1) ensures that

$$
\begin{aligned}
& \sigma_{\pi}\left(T^{-1} \Phi(A) T(x \otimes f)^{s}+(x \otimes f)^{s} T^{-1} \Phi(A) T\right) \\
= & \sigma_{\pi}\left(T\left[T^{-1} \Phi(A) T(x \otimes f)^{s}+(x \otimes f)^{s} T^{-1} \Phi(A) T\right] T^{-1}\right) \\
= & \sigma_{\pi}\left(\Phi(A) T(x \otimes f)^{s} T^{-1}+T(x \otimes f)^{s} T^{-1} \Phi(A)\right) \\
= & \sigma_{\pi}\left(A(x \otimes f)^{s}+(x \otimes f)^{s} A\right)
\end{aligned}
$$

Hence, by Lemma 3.3, we have $T^{-1} \Phi(A) T=A$ for all $A$ in $\mathcal{A}_{1}$, that is, $\Phi$ has the form (1) in the Theorem 1.2.

Similarly, one can show that $\Phi$ has the form (2) if (ii) of Claim 2.5 holds, completing the proof of Theorem 3.1 .

\section{REFERENCES}

[1] B. Aupetit, Spectrum-preserving linear mappings between Banach algebras or Jordan-Banach algebras, J. London Math. Soc., 62 (2000), 917-920.

[2] B. Aupetit and du T. Mouton H., Spectrum preserving linear mappings in Banach algebras, Studia Math., 109 (1994), 91-100.

[3] Z.-F. Bai and J.-C. Hou, Maps preserving zero-products or Jordan zero-products, Chinese Ann. Math. (A), 29(5) (2008), 663-670.

[4] J.-L. Cui and C.-K. Li, Maps preserving peripheral spectrum of Jordan products of operators, Operators and Matrices, 6 (2012), 129-146.

[5] J.-C. Hou, Rank-preserving linear maps $\mathcal{B}(X)$, Science in China., 32A (1989), 929-940.

[6] J.-C. Hou, C.-K. Li, N.-C. Wong, Jordan isomorphisms and maps preserving spectra of certain operator products, Studia Math., 184 (2008), 31-47.

[7] J.-C. Hou, C.-K. Li, N.-C. Wong, Maps Preserving the spectrum of generalized Jordan product of operators, Lin. Alg. Appl., 432 (2010), 1049-1069.

[8] A. A. Jafarian and A. R. Sourour, Spectrum-preserving linear maps, J. Funct. Anal., 66 (1986), $255-261$.

[9] I. Kaplansky, Algebraic and analytic aspects of operator algebras, CBMS Reg. Conf. Ser. in Math., vol.1, Amer. Math. Soc., Providence, 1970.

[10] I. Kaplansky, Infinite Abelian groups, U. of Michigan Press, Ann Arbor, Michigan, 1954.

[11] S. Lambert, A. Luttman and T. Tonev, Weakly peripherally-multiplicative mappings between uniform algebras, Contemp. Math., 435 (2007), 265-281.

[12] A. Luttman and T. Tonev, Uniform algebra isomorphisms and peripheral multiplicativity, Proc. Amer. Math. Soc., 135 (2007), 3589-3598.

[13] C. K. Li and N. K. Tsing, Linear preserver problems: A brief introduction and some special techniques, Lin. Alg. Appl., 162-164 (1992), 217-235.

[14] L. Molnár, Some characterizations of the automorphisms of $\mathcal{B}(H)$ and $\mathcal{C}(X)$, Proc. Amer. Math. Soc., 130 (2001), 111-120. 
[15] L. Molnár, Orthogonality preserving transformations on indefinite inner product space: Generalization of Uhlhorns version of Wigners theorem, J. Funct. Anal., 194 (2002), 248-262.

[16] T. Petek and P. Šemrl, Characterization of Jordan Homomorphisms on $M_{n}$ using preserver properties, Lin. Alg. Appl., 269 (1998), 33-46.

[17] T. Ransford, A Cartan theorem for Banach algebras, Proc. Amer. Math. Soc., 124 (1996), 243-247.

[18] N. V. Rao and A. K. Roy, Multiplicatively spectrum-preserving maps of function algebras, Proc. Amer. Math. Soc., 133 (2005), 1135-1142.

[19] N. V. Rao and A. K. Roy, Multiplicatively spectrum-preserving maps of function algebras.II, Proc. Edin. Math. Soc., 48 (2005), 219-229.

[20] A. R. Sourour, Invertibility preserving linear maps on $\mathcal{L}(X)$, Trans. Amer. Math. Soc., 348 (1996), 13-30.

[21] P. Š emrl, Non-linear commutativity preserving maps, Acta Sci. Math. (Szeged) 71 (2005), 781-819.

[22] M. Takeshi and H. Dai, A generalization of peripherally-multiplicatiove surjections between standard operator algebras, Central Eur. J. Math., 7(3) (2009), 479-486.

[23] T. Tonev and A. Luttman, Algebra isomorphisms between standard operator algebras, Studia Math., 191 (2009), 163-170.

[24] W. Zhang and J.-C. Hou, Maps preserving peripheral spectrum of Jordan semi-triple products of operators, Lin. Alg. Appl., 435 (2011), 1326-1335.

[25] W. Zhang and J.-C. Hou, Maps preserving peripheral spectrum of generalized products of operators, submitted.

(Wen Zhang) Department of Mathematics, Shanxi University, Taiyuan, Shanxi, 030006, P. R. China

E-mail address: wenzhang1314gw@163.com

(Jinchuan Hou) Department of Mathematics, Taiyuan University of Technology, Taiyuan 030024, P. R. of China; Department of Mathematics, Shanxi University, Taiyuan, Shanxi, 030006, P. R. China

E-mail address: houjinchuan@tyut.edu.cn

(Xiaofei Qi) Department of Mathematics, Shanxi University, Taiyuan, Shanxi, 030006, P. R. CHINA

E-mail address: qixf1980@126.com 